\title{
Nrf2 Regulates CHI3LI to Suppress Inflammation and Improve Post-Traumatic Osteoarthritis
}

\author{
Yang Song ${ }^{1,2}$ \\ Dake $\mathrm{Hao}^{3}$ \\ Huan Jiang ${ }^{4}$ \\ Mingguang Huang ${ }^{2}$ \\ Qingjun $\mathrm{Du}^{2}$ \\ Yi $\operatorname{Lin}^{2}$ \\ Fei Liu' \\ Bin Chen' \\ 'Division of Orthopaedics and \\ Traumatology, Department of \\ Orthopaedics, Nanfang Hospital, \\ Southern Medical University, Guangzhou, \\ 5I05I5, People's Republic of China; \\ ${ }^{2}$ Division of Traumatology and Joint, \\ Department of Orthopaedics, Shunde \\ Hospital, Southern Medical University, \\ Foshan, 528308, People's Republic of \\ China; ${ }^{3}$ Department of Surgery, School of \\ Medicine, University of California Davis, \\ Sacramento, CA, 95817, USA; \\ ${ }^{4}$ Department of Anesthesiology, Shunde \\ Hospital, Southern Medical University, \\ Foshan, 528308, People's Republic of \\ China
}

Introduction: Post-traumatic osteoarthritis (PTOA) is an inflammatory condition that occurs following mechanical joint trauma and that results in joint degeneration. This study sought to evaluate the regulatory function of nuclear factor erythroid 2-related factor 2 ( $\mathrm{Nrf} 2)$ in a murine model of anterior cruciate ligament transection (ACLT)-induced PTOA and in an in vitro model of synoviocyte inflammation induced by LPS treatment with the goal of exploring the role of chitinase 3-like-1 (CHI3L1) in this pathogenic context.

Methods: PTOA model mice were intra-articularly injected with Nrf2 overexpression lentiviral vector, and safranin O-fast green staining as well as the Osteoarthritis Research Society International (OARSI) Scoring System were used to evaluate the severity of cartilage damage. Protein expression in the synovial tissue was evaluated by Western blotting, immunohistochemical staining, and ELISA. Additionally, murine synoviocytes were infected with Nrf2 overexpression lentivirus and stimulated with LPS. The levels of inflammatory cytokines were detected by ELISA. ROS levels were measured using dihydroethidium (DHE) dye.

Results: We determined that the overexpression of Nrf2 was sufficient to reduce cartilage degradation in the context of PTOA in vivo, and we observed a significant decrease in the expression of matrix metalloproteinase 13 (MMP13) in the articular cartilage of samples from mice overexpressing Nrf2 relative to control mice. Synovial CHI3L1 expression and serum TNF- $\alpha$, IL- $1 \beta$, and IL-6 levels were reduced in animals overexpressing this transcription factor relative to PTOA model controls. Consistent with these findings, murine synoviocytes treated with LPS exhibited dose-dependent increases in ROS, TNF- $\alpha$, IL- $1 \beta$, IL-6, Nrf2, and CHI3L1 levels, whereas Nrf2 overexpression was sufficient to suppress these increases.

Conclusion: Our data indicated that Nrf2 negatively regulates CHI3L1, suggesting that this signaling axis may regulate PTOA progression and may thus be a viable therapeutic target in individuals affected by this condition.

Keywords: post-traumatic osteoarthritis, synovium, Nrf2, CHI3L1, oxidative stress, inflammation

\section{Introduction}

Post-traumatic osteoarthritis (PTOA) is a condition that arises as a consequence of trauma, resulting in pathological changes including articular cartilage ossification and degenerative chondrogenic hyperplasia, resulting in joint pain and dysfunction. ${ }^{1}$ While PTOA can affect individuals of any age, it is most common in young adults following traumatic injury or in the context of unbalanced or excessive load-bearing. ${ }^{2}$ As the ability of the articular cartilage to undergo selfrenewal is very limited, treatment options for PTOA remain unsatisfactory. ${ }^{3}$
Correspondence: Bin Chen Traumatology, Department of Orthopaedics, Nanfang Hospital, Southern Medical University, Guangzhou, 5I05I5, People's Republic of China Email chb@smu.edu.cn 
When reactive oxygen species (ROS) are produced at a rate faster than the rate at which the articular cartilage is able to clear them, this tissue is affected by oxidative stress. Oxidative stress levels are high in the cartilage in the context of PTOA, resulting in chronic inflammation, ${ }^{4}$ which can, in turn, drive synovial inflammation, intraarticular lesion formation, chondrocyte anabolic processed, and cartilage degeneration. ${ }^{5}$ Nuclear factor E2-related factor $2(\mathrm{Nrf} 2)$ is a transcription factor that serves as a primary regulator of cellular antioxidant responses. Under normal conditions in the absence of ongoing oxidative stress, Nrf2 is sequestered in the cytoplasm in an inactive state by Kelch-like ECH-associated protein 1 (Keap1). When oxidative stress occurs, however, Nrf2 dissociates from Keap1, becomes activated, and translocates to the nucleus wherein it is able to regulate antioxidant-related gene expression to protect cells against this deleterious form of stress. ${ }^{6}$ Osteoarthritis patients exhibit high levels of Nrf2 expression in the synovial tissues and cartilage, ${ }^{7,8}$ and the Nrf2/HO-1 signaling pathway has been shown to protect chondrocytes against hydrogen peroxide-induced oxidative damage and consequent apoptotic cell death. ${ }^{9}$ ROS generation and inflammation are closely linked processes that regulate one another, and which represent promising targets for PTOA treatment. ${ }^{10}$ Yan et $\mathrm{al}^{11}$ found that Nrf2 activation was sufficient to alleviate osteoarthritis via the inhibition of inflammasome activation.

Chitinase 3-like 1 (CHI3L1, also known as YKL-40) is a proinflammatory glycoprotein biomarker that has been linked to tissue remodeling and bone resorption. ${ }^{12} \mathrm{CHI} 3 \mathrm{~L} 1$ is secreted by macrophages, synoviocytes, and chondrocytes, ${ }^{13-16}$ and can be detected at increased levels in the synovial fluid and serum of patients with inflammatory conditions, in addition to correlating with the extent of joint degradation in rheumatoid arthritis (RA) patients. ${ }^{17}$ Inhibiting CHI3L1 has previously been shown to suppress inflammation and cortical bone degeneration in a murine osteomyelitis model system, ${ }^{18}$ while Kim et al ${ }^{19}$ similarly determined that knocking down this glycoprotein protected against hyperoxia-induced apoptotic death in human airway epithelial cells, whereas CHI3L1 overexpression increased the susceptibility of these cells to such apoptosis. As such, these prior data highlight CHI3L1 as an important regulator of inflammatory and oxidative stress responses. Its association with $\mathrm{Nrf} 2$, however, has yet to be defined.
Herein, we explored the relationship between Nrf2 and CHI3L1 by comparing the expression of these genes in synovial samples from PTOA model mice. We then explored the mechanistic basis for this relationship by conducting Nrf2 knockdown experiments both in vitro and in vivo.

\section{Materials and Methods}

\section{Animals}

C57BL/6 male mice (8-weeks-old) from Jiesijie Experimental Animal Co., Ltd (Shanghai, China) were housed in a climate-controlled facility $\left(23 \pm 1^{\circ} \mathrm{C} ; 50-60 \%\right.$ relative humidity; $12 \mathrm{~h}$ light/dark cycles). The Ethics Committee of Shunde Hospital approved these studies. This animal experiments was performed according to the guideline of Animal Care and Use Committee of Shunde Hospital and following internationally approved laboratory animal care principles published by the National Institute of Health.

\section{Nrf2 Overexpression}

The Nrf2 coding sequence (GI: 927028865) was synthesized, and both control and Nrf2 overexpression lentiviral vectors were prepared by Hanbio Biotechnology Co., LTD (Shanghai, China) at $3 \times 10^{8}$ transducing units (TU)/mL.

\section{PTOA Model Animal Treatment}

Mice were randomized into the following four groups ( $\mathrm{n}=5$ /group): a sham group, an ACLT group, an ACLT + Lenti-NC group, and an ACLT+Lenti-Nrf2 group. All ACLT model animals were anesthetized with $3 \%$ sodium pentobarbital (i.p.; Sigma-Aldrich, MO, USA). ACLT of the right knee was then conducted to cause joint instability, thereby inducing PTOA. In sham control mice, a skin and capsule incision was instead conducted. Animals in the lentiviral treatment groups were intra-articularly injected with $10 \mu \mathrm{L}$ of the appropriate negative control (Lenti-NC) or Nrf2 overexpression (Lenti-Nrf2) vectors on days 0,15 , 30 , and 45 after ACTL surgery, as reported previously. ${ }^{20}$ At eight weeks post-surgery, all mice were euthanized, and knee joints were collected for histological assessment.

\section{Histochemical and Immunohistochemical Staining}

Samples of paraffin-embedded tissue were cut to yield 4- $\mu \mathrm{m}$ thick sagittal sections which were then subjected to Hematoxylin and Eosin (HE), Masson's trichrome and Safranin O-Fast Green staining. Immunohistochemical 
staining for matrix metalloproteinase (MMP)-13 and alpha-smooth muscle actin ( $\alpha$-SMA) were conducted by deparaffinizing tissue sections, rehydrating them with an ethanol gradient, and conducting antigen retrieval by processing samples for $30 \mathrm{~min}$ with citrate buffer. Samples were then washed with PBS, treated with $3 \% \mathrm{H}_{2} \mathrm{O}_{2}$ to quench endogenous peroxidase activity, blocked for 1 $\mathrm{h}$ in $10 \%$ sheep serum at $37^{\circ} \mathrm{C}$, and treated overnight with anti-MMP13 (1/500, Abcam, USA) or anti- $\alpha$-SMA $(1 / 200, \mathrm{Abcam})$ at $4^{\circ} \mathrm{C}$. Sections were then probed with an appropriate HRP-linked secondary antibody (Abcam) for 45 min, washed with PBS, stained with the DAB substrate solution, and counterstained for $30 \mathrm{~s}$ with hematoxylin. A light microscope (Olympus Optical, Tokyo, Japan) was then used to image sections, which were evaluated using ImageJ. Two investigators blinded to experimental conditions calculated the percentage of staining positive area in six randomly selected fields of view for each articular cartilage section at a magnification of $\times 400$.

\section{Mouse OARSI Scoring}

In order to quantify the degree of cartilage degeneration, mouse cartilage degeneration was graded for Safranin $\mathrm{O} /$ Fast Green-stained knee sections using the Osteoarthritis Research Society International (OARSI) scoring system. A subjective score of $0-6$ was applied as previously described. $^{21}$ Grading was performed by three blinded observers. The three grades for each section were then averaged, and the data for mice in each group were collated. Higher scores were indicative of more serious cartilage damage.

\section{Immunofluorescent Staining}

Tissue sections were blocked for $1 \mathrm{~h}$ using 5\% goat serum at room temperature, and were then incubated overnight with anti-Nrf2 (1/500, Abcam) or anti-CHI3L1 (1/50, Santa Cruz, USA). Sections were then washed thrice with PBS and stained for $1 \mathrm{~h}$ with secondary goat anti-mouse AF488 (1/ 200, Invitrogen, USA) and rhodamine-conjugated goat antirabbit IgG (1/100, Abcam). Nuclei were then stained with DAPI, and cells were imaged via laser-scanning confocal microscopy (Olympus, Tokyo, Japan).

\section{Synovial Fibroblast Culture}

Minced synovial tissue from C57BL/6 mice was used to prepare primary murine synovial fibroblasts as in prior reports. $^{22}$ Briefly, these issues were digested for $3 \mathrm{~h}$ in serum-free DMEM containing $1 \mathrm{mg} / \mathrm{mL}$ of collagenase
(Roche, Meylan, France) at $37^{\circ} \mathrm{C}$. Cells were then washed twice with PBS prior to being cultured in DMEM containing $10 \%$ fetal calf serum (Life Technologies), $50 \mathrm{mg} / \mathrm{mL}$ L-glutamine, and penicillin/streptomycin in a humidified $5 \% \mathrm{CO}_{2}$ incubator. Synoviocytes used in the present study were obtained between passages 3 and 9, were positive for Vimentin immunofluorescent staining (anti-vimentin, 1:100, Abcam), and exhibited typical synovial fibroblast morphology. Lentiviral transduction was conducted by combining these cells $\left(1 \times 10^{5}\right)$ with Lenti-Nrf2 or Lenti$\mathrm{NC}$ at a multiplicity of infection (MOI) of 100 for $48 \mathrm{~h}$ in DMEM. Changes in Nrf2 protein expression were then evaluated via Western blotting.

\section{Intracellular ROS Assessment}

Murine synovial fibroblasts were treated for $24 \mathrm{~h}$ with a range of LPS concentrations, after which the dihydroethidium (DHE, Santa Cruz) dye was used to evaluate ROS production. Briefly, cells grown on glass coverslips were incubated in $\mathrm{HBSS}$ (Gibco) containing $\mathrm{CaCl}_{2}$, $\mathrm{MgCl}_{2}$, and DHE $(10 \mu \mathrm{mol} / \mathrm{L})$ for $30 \mathrm{~min}$ at $37^{\circ} \mathrm{C}$ in the dark. Flow cytometry was then used to evaluate ROS production based on median fluorescence intensity values for 10,000 cells.

\section{ELISAs}

On day 7 post-surgery, samples of blood were obtained from experimental animals. Blood was spun for $15 \mathrm{~min}$ at $3000 \mathrm{rpm}$ at $4^{\circ} \mathrm{C}$ to yield serum. Levels of TNF- $\alpha$ (\#MTA00B), IL-1ß (\#MLB00C), and IL-6 (\#M6000B) in serum and in samples of supernatants from cultured synovial fibroblasts were measured with commercial ELISA kits (R\&D Systems, MN, USA) based on provided directions. The absorbance of each standard and sample was measured at $450 \mathrm{~nm}$. A standard concentration gradient was used as a standard curve.

\section{Western Blotting}

RIPA buffer supplemented with protease inhibitor cocktail (Pierce, IL, USA) was used to lyse cells, after which nuclear and cytoplasmic proteins were isolated with a commercially available nuclear and cytoplasmic extraction kit (Thermo Fisher Inc., IL, USA) based on provided instructions. A BCA assay (Beyotime Biotechnology, China) was then used to measure protein levels, and equal amounts of protein $(40 \mu \mathrm{g})$ per sample were separated via 10\% SDS-PAGE and transferred onto PVDF membranes (Millipore, Germany) that were then blocked using 5\% non-fat milk in TBST for 1 

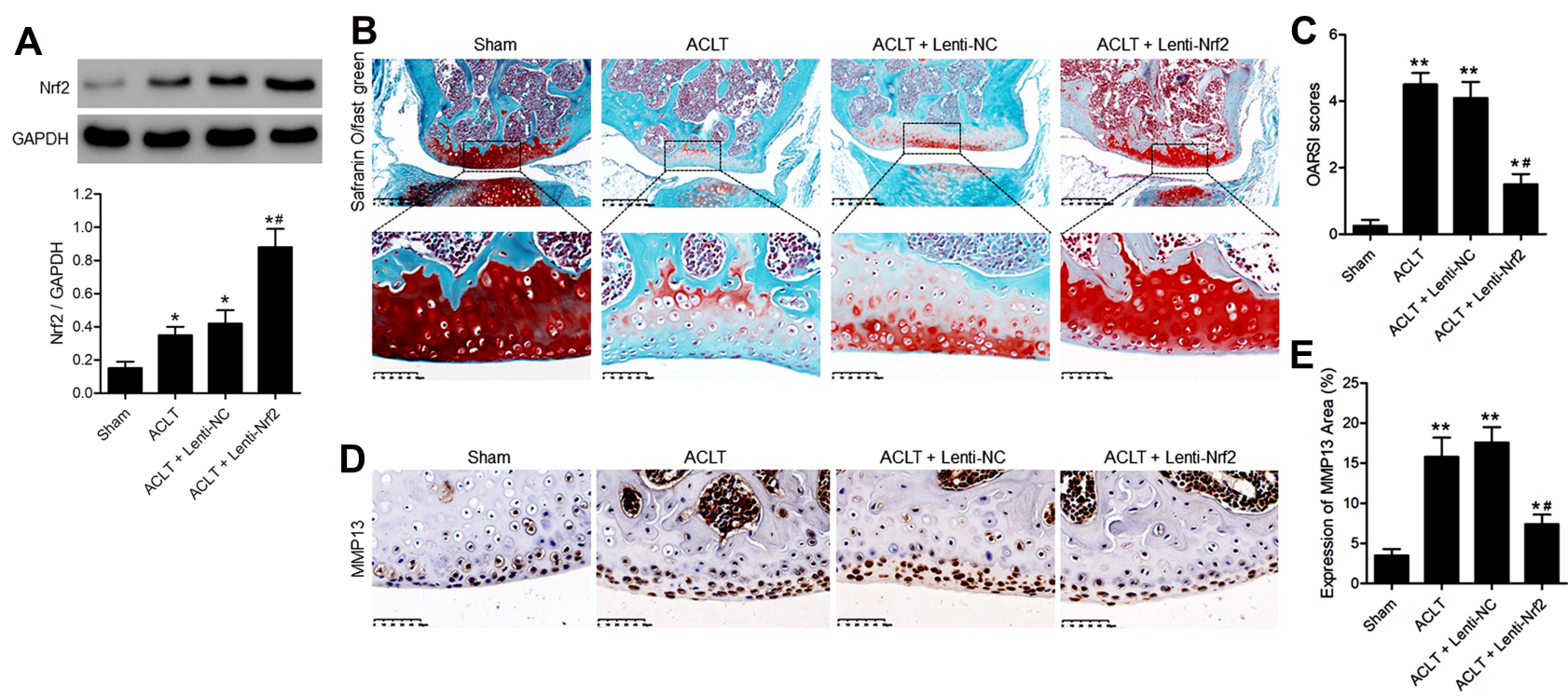

Figure I Overexpressing Nrf2 inhibits articular cartilage degeneration in a murine model of ACLT-induced PTOA. On day 3 post-ACLT, the affected knee joints of mice were injected with a lentivirus encoding a control or Nrf2 overexpression vector (Lenti-NC and Lenti-Nrf2, respectively). At 8 weeks post-ACLT, all animals were euthanized. Sham-operated animals served as negative controls. (A) Nrf2 protein levels in knee joints of mice were assessed by Western blotting with GAPDH as a normalization control. (B) Sagittal tibial articular cartilage sections were subjected to safranin O-fast green staining. Scale bar $=200 \mu \mathrm{m}$ or $50 \mu \mathrm{m}$. (C) the OARSI scoring system was used to grade mouse cartilage degeneration. (D) Cartilage expression of MMPI3 was evaluated via immunohistochemistry. Scale bar $=50 \mu \mathrm{m}$. (E) The MMPI3positive cartilage area was quantified as a fraction of overall cartilage area. Data are means \pm SD. $n=5 / g r o u p$. * $p<0.05$, ** $p<0.01$ vs Sham. \#p<0.05 vs ACLT model group.

h. Next, blots were probed overnight with anti-Nrf2 (1:1000, Abcam), anti-CHI3L1 (1:200, Santa Cruz), anti-LaminB (1:1000, Sigma), anti- $\alpha$-tubulin (1:1000, Santa Cruz), or antiGAPDH $(1: 1000$, Santa Cruz $)$ at $4^{\circ} \mathrm{C}$. Blots were then washed using TBST and probed with an appropriate secondary antibody for $2 \mathrm{~h}$ at room temperature, after with the ECL Plus reagent (Amersham Biosciences, Little Chalfont, UK) and autoradiographic film were used to detect protein bands. A gel image analysis system (BioRad, CA, USA) was used for densitometric analyses of developed blots.

\section{Statistical Analysis}

Data are means $\pm \mathrm{SD}$, and were compared through Student's t-tests or one-way ANOVAs with Tukey's post hoc test, as appropriate. $\mathrm{P}<0.05$ was the significance threshold for this study.

\section{Results}

\section{Overexpressing Nrf2 Prevents Articular Cartilage Degeneration in a Murine Model of PTOA}

Western blotting was performed to determine the expression of $\mathrm{Nrf} 2$ in knee joints of mice. Compared with that in the sham group, the expression of Nrf2 in ACLT group was increased. Notably, the expression of Nrf2 in the Nrf2 overexpression $($ ACLT+Lenti-Nrf2) group was increased significantly compared with that in the ACLT group (Figure 1A). Safranin $\mathrm{O} /$ fast green staining was used to evaluate articular cartilage structures in our experimental mice. In sham control animals, the articular cartilage was intact with a smooth surface and uniform organization (Figure 1B). In contrast, animals in the ACLT and ACLT+Lenti-NC groups exhibited a reduction in safranin $\mathrm{O}$ staining consistent with a loss of articular cartilage. However, ACLT+Lenti-Nrf2 treatment significantly decreased the severity of articular cartilage degeneration in these PTOA model animals. The ACLT+Lenti-Nrf2 group also exhibited significantly lower cartilage OARSI grade scores relative to the ACLT and ACLT+Lenti-NC groups (Figure 1C). We then conducted immunohistochemical staining for MMP13 in synovial tissue samples from these mice (Figure 1D), revealing significant increases in MMP13-positive cartilage area in ACLT and ACLT+Lenti-NC group animals relative to sham controls, whereas this area was significantly decreased in animals in the ACLT+Lenti-Nrf2 group relative to those in the ACLT model group (Figure 1E).

\section{Overexpressing Nrf2 Prevents Synovial Fibrosis and Inflammation in PTOA Model Mice}

Masson's trichrome staining was next conducted to assess synovial fibrosis in these mice at 8 weeks post-surgery. This analysis revealed that Nrf2 overexpression markedly 

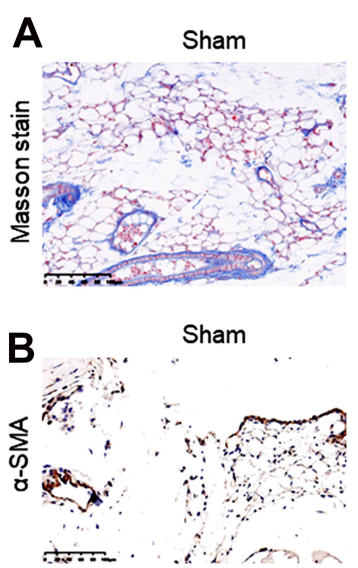

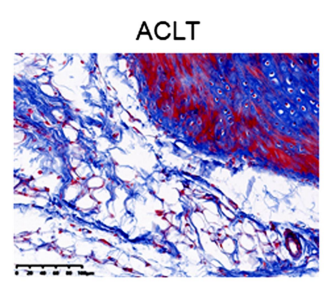

ACLT

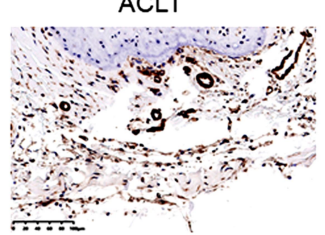

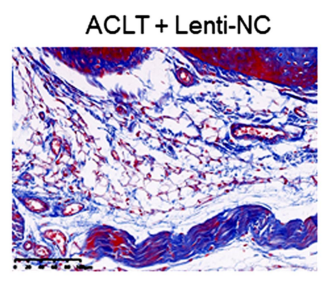

$A C L T+$ Lenti-NC

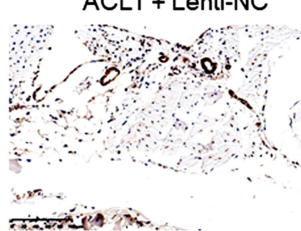

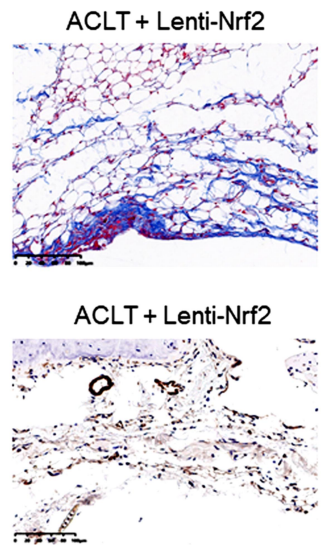

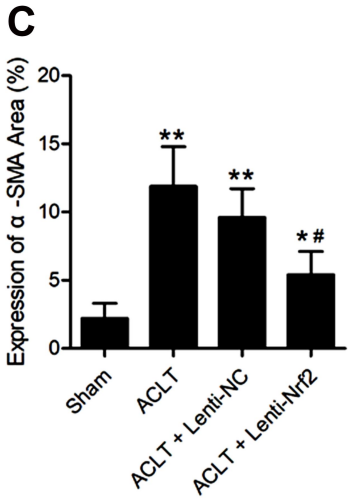

Figure 2 Overexpressing Nrf2 suppresses synovial fibrosis in a murine model of PTOA. (A) Masson's staining of synovial samples from mice in the indicated treatment groups was conducted. Scale bar $=100 \mu \mathrm{m}$. (B) $\alpha$-SMA expression in synovial samples was evaluated via immunohistochemistry. Scale bar $=100 \mu \mathrm{m}$. (C) The $\alpha$-SMA-positive synovial area was quantified as a fraction of overall synovial area. Data are means \pm SD. $n=5 /$ group. ${ }^{*}<0.05,{ }^{* *} p<0.01$ vs Sham. \#p $<0.05$ vs ACLT model group.

suppressed ACLT-induced increases in the fibrotic area (Figure 2A). Immunohistochemical staining also confirmed that the expression of the fibrosis marker $\alpha$-SMA was significantly increased in the ACLT group in comparison with the Sham group, while overexpression of Nrf2 in the ACLT+Lenti-Nrf2 group appeared to reduce their expression against the ACLT group (Figure 2B and C). HE staining displayed pathological changes among groups, and immunofluorescent staining of synovial tissues revealed that ACLT surgery induced Nrf2 translocation to the nucleus, and this localization was increased in mice injected with Lenti-Nrf2. We also observed significantly higher CHI3L1 levels in PTOA model mice, and found that this protein colocalized with Nrf2 in synovial tissue samples (Figure 3 ). We additionally collected serum samples from experimental mice on day 7 post-surgery and measured TNF- $\alpha$, IL- $1 \beta$, and IL-6 levels therein. This analysis revealed that ACLT surgery increased the circulating levels of all three of these cytokines, whereas Lenti-Nrf2 administration markedly
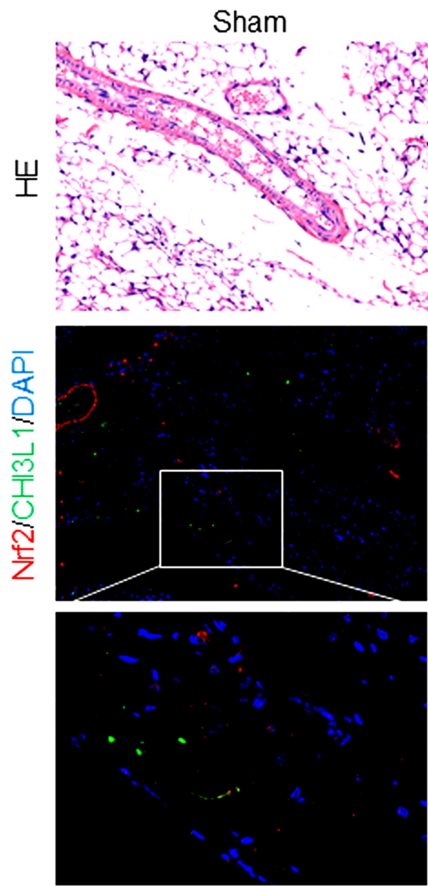
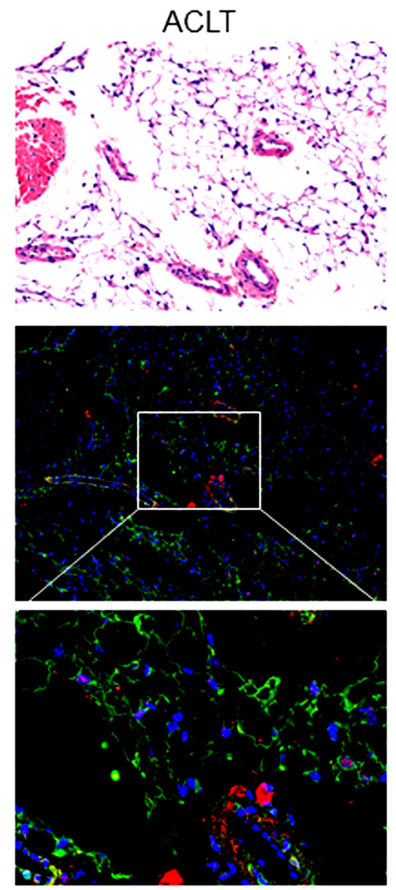
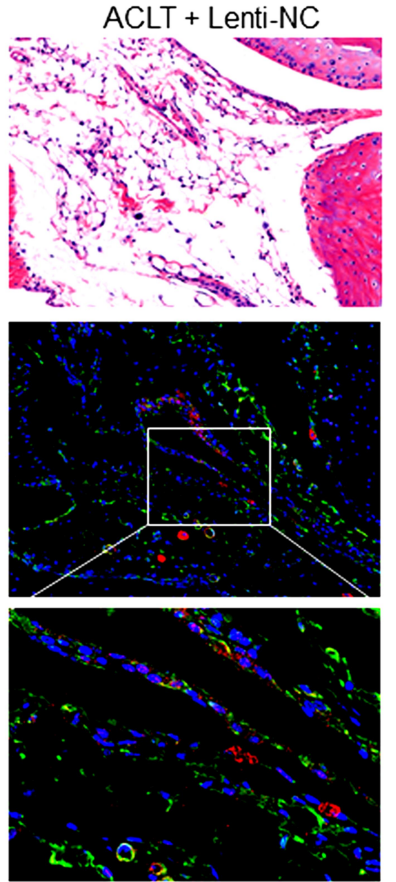

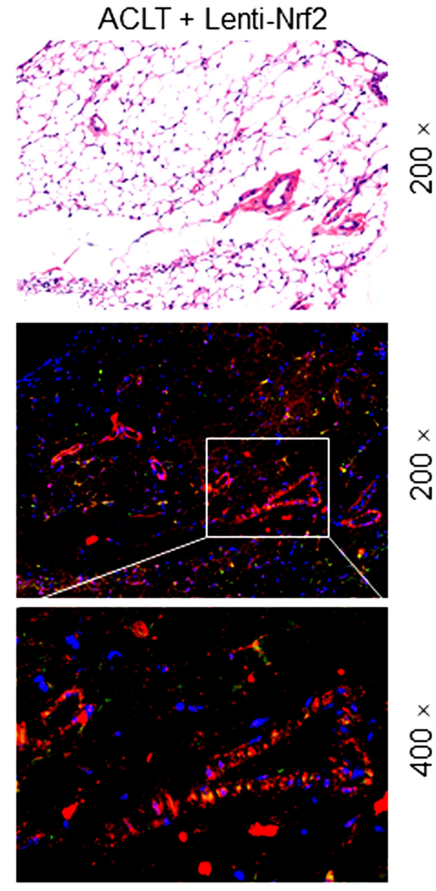

Figure $3 \mathrm{HE}$ and immunofluorescence staining of the synovium in PTOA model mice. Synovial samples were stained for HE and immunofluorescence of Nrf2 (red) and CHI3LI (green), with DAPI (blue) being used for nuclear counterstaining. The magnification is $200 \times$ or $400 \times$. 

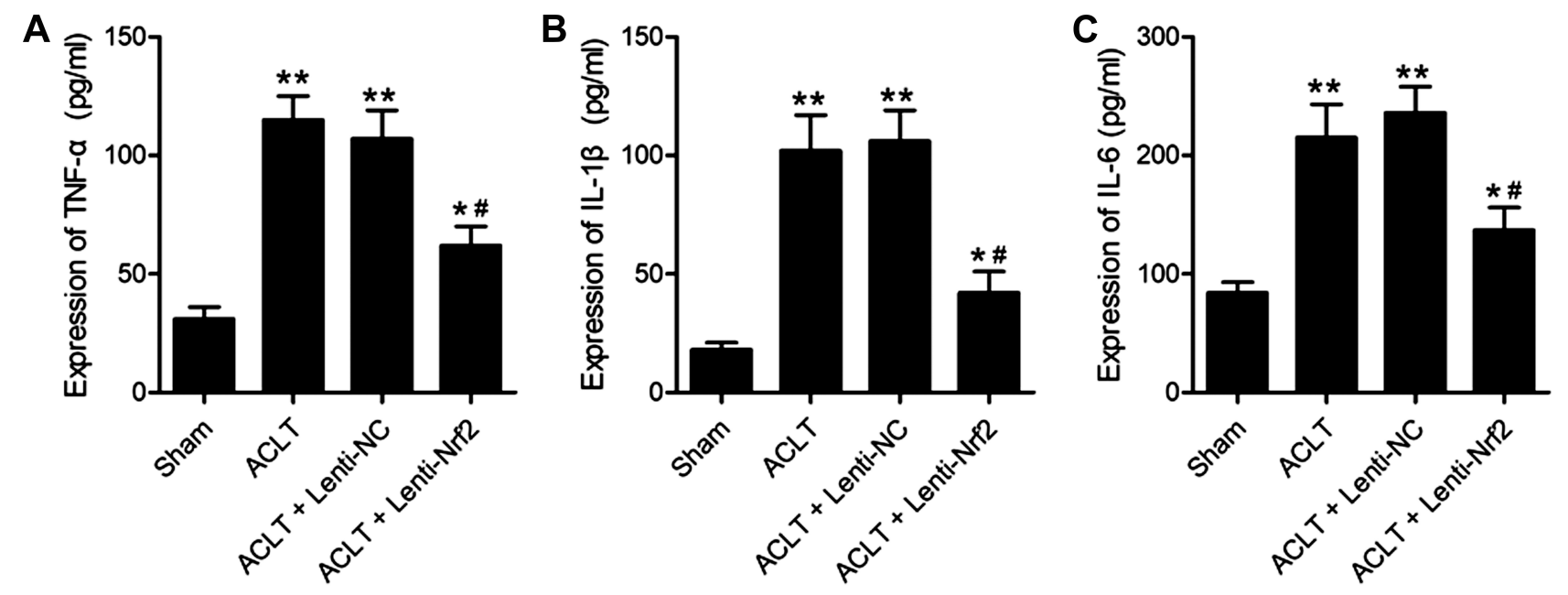

Figure 4 Overexpressing Nrf2 suppresses synovial inflammation in a murine model of PTOA. Samples of blood were conducted on day-7 post-ACLT or sham surgery, and ELISAs were conducted to measure TNF- $\alpha(\mathbf{A})$, IL-I $\beta$ (B), and IL-6 (C) levels in isolated serum. Data are means \pm SD. $n=5 / g r o u p . ~ * p<0.05$, **p<0.0I vs Sham. \#p<0.05 vs ACLT model group.

suppressed their expression relative to ACLT model control mice (Figure $4 \mathrm{~A}-\mathrm{C}$ ), suggesting that Nrf2 can inhibit inflammatory mediator expression.

\section{LPS Treatment of Murine Synoviocytes Induces Inflammation and ROS \\ Production}

Figure 5A displays the immunofluorescence-based identification of synoviocytes. We treated murine synoviocytes with LPS $(0,50,100$, or $200 \mathrm{ng} / \mathrm{mL})$ for $24 \mathrm{~h}$. DHE staining of these cells then revealed that ROS levels were significantly increased in a dose-dependent fashion upon LPS treatment (Figure 5B and C), as were TNF- $\alpha$, IL-1 $\beta$, and IL-6 levels (Figure 5D-F). We then evaluated Nrf2 and CHI3L1 protein levels in these synoviocytes via Western blotting, revealing both to be upregulated by LPS in a dose-dependent fashion (Figure 5G and $\mathrm{H}$ ). In particular, extremely significant increases in all the above indicators were observed in cells treated with LPS concentrations $\geq 100 \mathrm{ng} / \mathrm{mL}$, as compared to cells not stimulated with LPS. As such, $100 \mathrm{ng} / \mathrm{mL}$ of LPS was selected as the concentration used in subsequent experiments.

\section{Overexpressing Nrf2 Suppresses}

\section{LPS-Induced Synoviocyte CHI3LI Protein} Expression, Inflammation, and ROS

\section{Production}

Lastly, we explored the functional role of Nrf2 in murine synoviocytes by transducing these cells for $48 \mathrm{~h}$ with Lenti-
$\mathrm{NC}$ or Lenti-Nrf2 and then treating them for an additional 24 $\mathrm{h}$ with LPS $(100 \mathrm{ng} / \mathrm{mL})$. Western blotting revealed that nuclear Nrf2 levels were significantly higher in Nrf2 overexpressing cells relative to those transduced with Lenti-NC, whereas CHI3L1 levels were significantly reduced upon Lenti-Nrf2 infection (Figure 6A and B). Following LPS treatment, ROS levels in these cells were assessed via DHE staining (Figure 6C), revealing significant reductions in ROS levels in cells overexpressing Nrf2 (Figure 6D). We additionally evaluated inflammatory cytokine levels in supernatants collected from these cells following LPS treatment, revealing that TNF- $\alpha$ (Figure 6E), IL-1 $\beta$ (Figure 6F), and IL-6 (Figure 6G) levels were all significantly decreased by Nrf2 overexpression compared to Lenti-NC transduction.

\section{Discussion}

PTOA is a form of osteoarthritis (OA), a chronic disease that can cause synovial joint pain and even disability in severe cases. ${ }^{23}$ The knee is the most common site of traumatic synovitis in the body. ${ }^{24}$ Herein, we utilized a murine model of PTOA via conducting ACLT of the right knee joint. At 8 weeks post-ACLT, articular cartilage degeneration was clearly evident in the operated knee of these mice. MMP13 is a major driver of cartilage degradation, ${ }^{25}$ and we found that the ACLT procedure was associated with a significant increase in MMP13 expression in the articular cartilage of these mice relative to sham-operated controls. We also found that serum TNF- $\alpha$, IL- $1 \beta$, and IL-6 levels were higher in ACLT model mice. Together these data are 

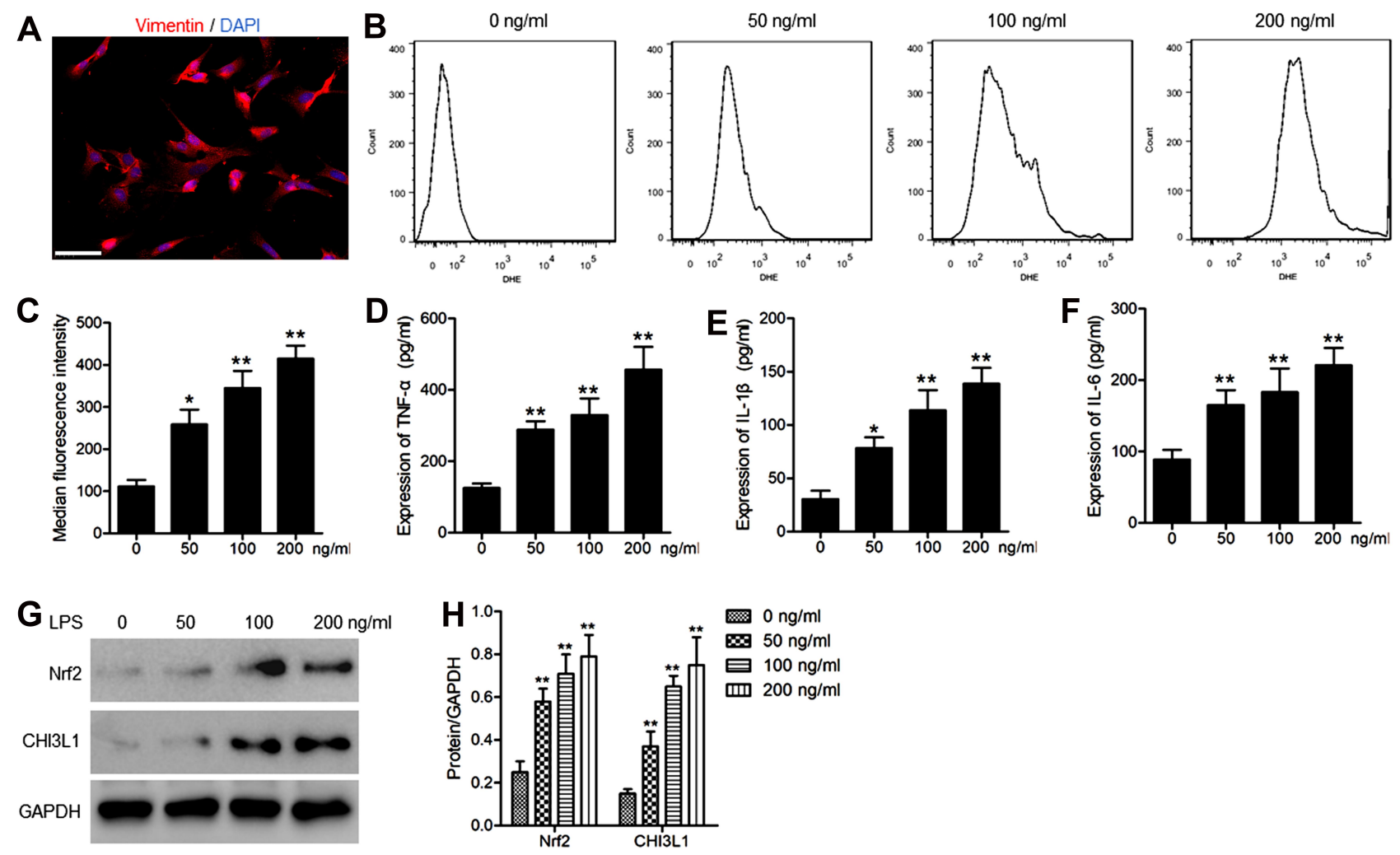

Figure 5 LPS treatment of murine synoviocytes induces inflammation and ROS production. (A) Representative immunofluorescence images used to identify synoviocytes. Images of vimentin staining (red). Nuclei were counterstained with DAPI (blue). Scale bar $=50 \mu \mathrm{m}$. (B) ROS levels in cells treated with a range of LPS concentrations for 24 $\mathrm{h}$ were evaluated via flow cytometry. (C) Median fluorescent intensity values were used to quantify ROS data. ELISAs were used to measure supernatant TNF- $\alpha$ (D), IL-I $\beta$ (E), and IL-6 (F) concentrations. (G) Western blotting was used to assess Nrf2 and CHI3LI protein levels, (H) with GAPDH as a normalization control. Data are means \pm SD from three technical replicates. ${ }^{*} \mathrm{p}<0.05,{ }^{* *} \mathrm{p}<0.01$ vs untreated.

consistent with clinical signs of osteoarthritis such as synovitis and cartilage degeneration. ${ }^{1,2,23}$

The synovium plays a key role in maintaining joint homeostasis, lubricating the joint and facilitating the flow of synovial fluid therein. ${ }^{26}$ Herein, we observed clear signs of synovial fibrosis in ACLT model mice, consistent with the impairment of synovial function. Coleman et $\mathrm{al}^{27}$ determined that intra-articular $\mathrm{N}$-acetylcysteine (NAC) injection can effectively prevent PTOA via suppressing oxidative stress in model experimental systems. As a key regulator of oxidative stress responses, $\mathrm{Nrf} 2$ is primarily localized to the cytoplasm under normal physiological conditions, but rapidly localizes to the nucleus in response to oxidative stress whereupon it can promote the upregulation of antioxidant stress response protein. ${ }^{6,9} \mathrm{Su}$ et $\mathrm{al}^{28}$ showed that the activation of p62/Nrf2 signaling was sufficient to inhibit ROS production, thus alleviating bone erosion in rheumatoid arthritis. Moreover, Lal et al ${ }^{29}$ confirmed that Nrf2/HO-1 signaling pathway activation improved arthritis in rats. Our data indicated that $\mathrm{Nrf} 2$ activation occurs in the synovial tissue of ACLT model mice, and this activity was enhanced by Nrf2 overexpression. In addition, Nrf2 overexpression inhibited fibrosis, cartilage degeneration, and systemic inflammation in these ACLT model mice, indicating that activation of Nrf2 is beneficial to the development of PTAO. In our study, CHI3L1 expression was also increased in the synovium of mice following ACLT, whereas Nrf2 overexpression suppressed this CHI3L1 upregulation. CHI3L1 has been shown to be a proinflammatory biomarker that is reflective of the extent of synovial inflammation and cartilage degradation. ${ }^{17,30}$ These data indicate that Nrf2 can downregulate $\mathrm{CHI} 3 \mathrm{~L} 1$ and thereby suppress inflammation in the context of PTOA.

ROS have been identified as key regulators of inflammation, controlling oxidative stress and antioxidant enzyme activity and thereby promoting an increase in inflammatory activity. ${ }^{31}$ We found that ROS and inflammatory cytokine levels were increased in a dose-dependent manner in murine synoviocytes treated with LPS, and we found Nrf2 and CHI3L1 expression to be similarly increased. We then treated Nrf2-overexpressing synovial 


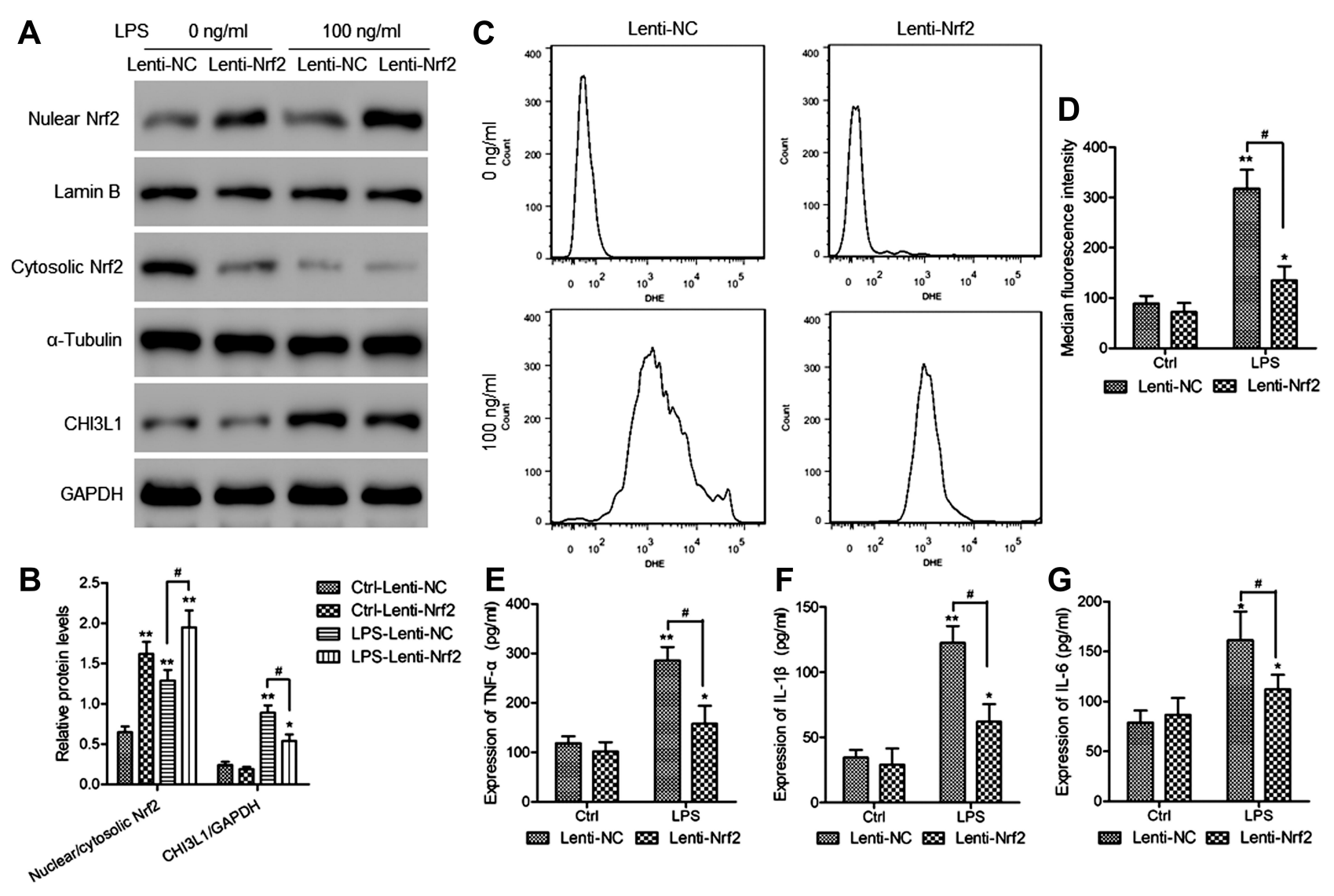

Figure 6 Overexpressing Nrf2 suppresses LPS-induced synoviocyte CHI3LI protein expression, inflammation, and ROS production. Murine synoviocytes were infected for $48 \mathrm{~h}$ with Lenti-NC or Lenti-Nrf2 vectors, after which they were either left untreated (Ctrl) or were treated for $24 \mathrm{~h} \mathrm{with} \mathrm{LPS} \mathrm{(I00} \mathrm{ng/mL).} \mathrm{(A)} \mathrm{Levels} \mathrm{of} \mathrm{Nrf2} \mathrm{in} \mathrm{cytosolic}$ and nuclear extracts were assessed via Western blotting, as were $\mathrm{CHI} 3 \mathrm{LI}$ levels in total cell lysates from synoviocytes in the indicated treatment groups. (B) Levels of nuclear Nrf2 were normalized against cytoplasmic Nrf2 levels, whereas GAPDH was used when normalizing CHI3LI expression. (C) ROS production in synoviocytes was evaluated via flow cytometry, (D) with median fluorescence intensity values being used to quantify these results. ELISAs were used to measure supernatant TNF- $\alpha$ (E), IL-I $\beta$ $(\mathbf{F})$, and IL-6 (G) levels. Data are means \pm SD from three technical replicates. ${ }^{*} p<0.05,{ }^{* *} p<0.01$ vs Lenti-NC infected control cells $(C$ trl-Lenti-NC). \#p<0.05 vs Lenti-Nrf2 infected control cells (LPS-Lenti-NC).

fibroblasts with LPS in order to explore the association between CHI3L1 and Nrf2 in this pathogenic context. We found that CHI3L1 expression was significantly reduced in cells overexpressing this transcription factor relative to cells transduced with a control lentivirus. ROS can trigger Nrf2 activation, which can suppress oxidative stress and thereby inhibit inflammation. ${ }^{32}$ Our data indicate that Nrf2 overexpression may suppress CHI3L1 expression and ROS production, thereby inhibiting inflammatory responses.

\section{Conclusion}

Together, our data indicate that Nrf2 and CHI3L1 are expressed at high levels in the murine PTOA model system. Importantly, we determined that Nrf2 overexpression downregulates CHI3L1 expression and suppresses inflammation in vivo and in vitro. Nrf2 may therefore be a viable target for therapeutic efforts aimed at treating or preventing PTOA through mechanisms linked to the regulation of CHI3L1. However, one noteworthy limitation of this study is that the mechanisms and pathways whereby Nrf2 affects the cartilage and synoviocytes were not analyzed, and future research will be required to validate and expand upon the findings of this study in order to fully clarify the mechanisms and pathways underlying this Nrf2mediated activity and the relationship between Nrf2 and CHI3L1 in the synovium and articular cartilage in the context of PTOA.

\section{Funding}

The authors received no funding for this work.

\section{Disclosure}

The authors declare no conflicts of interest. 


\section{References}

1. Whittaker JL, Roos EM. Infographic. Risk profile for sport-related post-traumatic knee osteoarthritis. $B r \quad J$ Sports Med. 2020;54 (6):362-363.

2. Chery DR, Han B, Li Q, et al. Early changes in cartilage pericellular matrix micromechanobiology portend the onset of post-traumatic osteoarthritis. Acta biomaterialia. 2020;111:267-278. doi:10.1016/j. actbio.2020.05.005

3. Dai C, Kuang Z. An invited commentary on: lingering risk: a meta-analysis of outcomes following primary total knee arthroplasty for patients with post-traumatic arthritis. Int $j$ Surgery. 2020;78:103. doi:10.1016/j.ijsu.2020.04.036

4. Liang R, Zhao J, Li B, et al. Implantable and degradable antioxidant poly(epsilon-caprolactone)-lignin nanofiber membrane for effective osteoarthritis treatment. Biomaterials. 2020;230:119601. doi:10.1016/j.biomaterials.2019.119601

5. Leimer EM, Tanenbaum LM, Nettles DL, et al. Amino Acid profile of synovial fluid following intra-articular ankle fracture. Foot Ankle Int. 2018;39(10):1169-1177. doi:10.1177/1071100718786163

6. Dodson M, de la Vega MR, Cholanians AB, Schmidlin CJ, Chapman E, Zhang DD. Modulating NRF2 in Disease: timing Is Everything. Annu Rev Pharmacol Toxicol. 2019;59:555-575. doi:10.1146/annurev-pharmtox-010818-021856

7. Khan NM, Ahmad I, Haqqi TM. Nrf2/ARE pathway attenuates oxidative and apoptotic response in human osteoarthritis chondrocytes by activating ERK1/2/ELK1-P70S6K-P90RSK signaling axis. Free Radic Biol Med. 2018;116:159-171. doi:10.1016/j. freeradbiomed.2018.01.013

8. Chen $\mathrm{Z}$, Zhong $\mathrm{H}$, Wei J, et al. Inhibition of Nrf2/HO-1 signaling leads to increased activation of the NLRP3 inflammasome in osteoarthritis. Arthritis Res Ther. 2019;21(1):300. doi:10.1186/ s13075-019-2085-6

9. Kim EN, Lee HS, Jeong GS. Cudratricusxanthone O Inhibits H2O2-Induced Cell Damage by Activating Nrf2/HO-1 Pathway in Human Chondrocytes. Antioxidants. 2020;9(9):788. doi:10.3390/ antiox 9090788

10. Ansari MY, Ahmad N, Haqqi TM. Oxidative stress and inflammation in osteoarthritis pathogenesis: role of polyphenols. Biomed Pharmacother. 2020;129:110452. doi:10.1016/j.biopha.2020.110452

11. Yan Z, Qi W, Zhan J, et al. Activating Nrf2 signalling alleviates osteoarthritis development by inhibiting inflammasome activation. J Cell Mol Med. 2020;24(22):13046-13057. doi:10.1111/jcmm.15905

12. Steinke J, Samietz S, Friedrich N, et al. Associations of plasma YKL-40 concentrations with heel ultrasound parameters and bone turnover markers in the general adult population. Bone. 2020;141:115675. doi:10.1016/j.bone.2020.115675

13. Kim EG, Kim MN, Hong JY, et al. Chitinase 3-Like 1 Contributes to Food Allergy via M2 Macrophage Polarization. Allergy Asthma Immunol Res. 2020;12(6):1012-1028. doi:10.4168/aair.202 0.12.6.1012

14. Holst CB, Christensen IJ, Skjoth-Rasmussen J, Hamerlik P, Poulsen HS, Johansen JS. Systemic Immune Modulation in Gliomas: prognostic Value of Plasma IL-6, YKL-40, and Genetic Variation in YKL-40. Front Oncol. 2020;10:478. doi:10.3389/ fonc. 2020.00478

15. Wang $\mathrm{R}$, Xu C, Zhong $\mathrm{H}$, et al. Inflammatory-sensitive CHI3L1 protects nucleus pulposus via AKT3 signaling during intervertebral disc degeneration. FASEB J. 2020;34(3):3554-3569. doi:10.1096/ fj.201902096R

16. Kazakova MH, Batalov AZ, Mateva NG, Kolarov ZG, Sarafian VS YKL-40 and cytokines - A new diagnostic constellation in rheumatoid arthritis? Folia Med (Plovdiv). 2017;59(1):37-42. doi:10.1515/ folmed-2017-0013
17. Karalilova R, Kazakova M, Batalov A, Sarafian V. Correlation between protein YKL-40 and ultrasonographic findings in active knee osteoarthritis. Med Ultrason. 2018;1(1):57-63. doi:10.11152/ mu-1247

18. Chen X, Jiao J, He X, et al. CHI3L1 regulation of inflammation and the effects on osteogenesis in a Staphylococcus aureus-induced murine model of osteomyelitis. FEBS J. 2017;284(11):1738-1747. doi:10.1111/febs. 14082

19. Kim MN, Lee KE, Hong JY, et al. Involvement of the MAPK and PI3K pathways in chitinase 3-like 1-regulated hyperoxia-induced airway epithelial cell death. Biochem Biophys Res Commun. 2012;421(4):790-796. doi:10.1016/j.bbrc.2012.04.085

20. Zheng G, Zhan Y, Li X, et al. TFEB, a potential therapeutic target for osteoarthritis via autophagy regulation. Cell Death Dis. 2018;9 (9):858. doi:10.1038/s41419-018-0909-y

21. Schott EM, Farnsworth CW, Grier A, et al. Targeting the gut microbiome to treat the osteoarthritis of obesity. JCI Insight. 2018;3(8). doi:10.1172/jci.insight.95997.

22. Gao B, Calhoun K, Fang D. The proinflammatory cytokines IL-1beta and TNF-alpha induce the expression of Synoviolin, an E3 ubiquitin ligase, in mouse synovial fibroblasts via the Erk1/2-ETS1 pathway. Arthritis Res Ther. 2006;8(6):R172. doi:10.1186/ar2081

23. Lai-Zhao Y, Pitchers KK, Appleton CT. Transient anabolic effects of synovium in early post-traumatic osteoarthritis: a novel ex vivo joint tissue co-culture system for investigating synovium-chondrocyte interactions. Osteoarthritis Cartilage OARS. 2021;29(7):1060-1070. doi:10.1016/j.joca.2021.03.010

24. Bailey KN, Furman BD, Zeitlin J, et al. Intra-articular depletion of macrophages increases acute synovitis and alters macrophage polarity in the injured mouse knee. Osteoarthritis Cartilage OARS. 2020;28(5):626-638. doi:10.1016/j.joca.2020.01.015

25. Takahashi A, de Andres MC, Hashimoto K, et al. DNA methylation of the RUNX2 P1 promoter mediates MMP13 transcription in chondrocytes. Sci Rep. 2017;7(1):7771. doi:10.1038/s41598-01708418-8

26. Bao J, Yan W, Xu K, et al. Oleanolic Acid Decreases IL-1betaInduced Activation of Fibroblast-Like Synoviocytes via the SIRT3-NF-kappaB Axis in Osteoarthritis. Oxid Med Cell Longev. 2020;2020:7517219. doi:10.1155/2020/7517219

27. Coleman MC, Goetz JE, Brouillette MJ, et al. Targeting mitochondrial responses to intra-articular fracture to prevent posttraumatic osteoarthritis. Sci Transl Med. 2018;10(427):eaan5372. doi:10.1126/ scitranslmed.aan5372

28. Su X, Guo W, Yuan B, et al. Artesunate attenuates bone erosion in rheumatoid arthritis by suppressing reactive oxygen species via activating p62/Nrf2 signaling. Biomed Pharmacother. 2021;137:111382. doi:10.1016/j.biopha.2021.111382

29. Lal R, Dhaliwal J, Dhaliwal N, Dharavath RN, Chopra K. Activation of the Nrf2/HO-1 signaling pathway by dimethyl fumarate ameliorates complete Freund's adjuvant-induced arthritis in rats. Eur J Pharmacol. 2021;899:174044. doi:10.1016/j.ejphar.2021.174044

30. Dundar U, Asik G, Ulasli AM, et al. Assessment of pulsed electromagnetic field therapy with Serum YKL-40 and ultrasonography in patients with knee osteoarthritis. Int $J$ Rheum Dis. 2016;19 (3):287-293. doi:10.1111/1756-185X.12565

31. Kim GY, Jeong H, Yoon HY, et al. Anti-inflammatory mechanisms of suppressors of cytokine signaling target ROS via NRF-2/thioredoxin induction and inflammasome activation in macrophages. Bmb Rep. 2020;53(12):640-645. doi:10.5483/BMBRep.2020.53.12.161

32. Kahroba H, Ramezani B, Maadi H, Sadeghi MR, Jaberie H, Ramezani F. The role of Nrf2 in Neural stem/Progenitors cells: from maintaining stemness and self-renewal to promoting differentiation capability and facilitating therapeutic application in neurodegenerative disease. Ageing Res Rev. 2020;2:101211. 


\section{Publish your work in this journal}

The Journal of Inflammation Research is an international, peerreviewed open-access journal that welcomes laboratory and clinical findings on the molecular basis, cell biology and pharmacology of inflammation including original research, reviews, symposium reports, hypothesis formation and commentaries on: acute/chronic inflammation; mediators of inflammation; cellular processes; molecular mechanisms; pharmacology and novel anti-inflammatory drugs; clinical conditions involving inflammation. The manuscript management system is completely online and includes a very quick and fair peerreview system. Visit http://www.dovepress.com/testimonials.php to read real quotes from published authors.

Submit your manuscript here: https://www.dovepress.com/journal-of-inflammation-research-journal 